\title{
AN INITIAL DESIGN FRAMEWORK FOR VIRTUAL HISTORIC DUBLIN
}

\author{
M Murphy ${ }^{1}, \mathrm{~S} \mathrm{Pavia}^{1}$, J Cahill ${ }^{2}$, S Lenihan ${ }^{2}, \mathrm{~A} \mathrm{Corns}^{3}$, \\ ${ }^{1}$ Trinity College Dublin, maurice.m.murphy@gmail.com, pavias@tcd.ie \\ ${ }^{2}$ Office of Public Works, John.Cahill@OPW.ie, shane.lenihan@opw.ie \\ ${ }^{3}$ Discovery Programme, anthony@discoveryprogramme.ie
}

\section{Commission VI, WG VI/4}

KEY WORDS: Historic City Modelling, GIS, HBIM, Laser Scanning, 3D Modelling

\begin{abstract}
:
As a single coherent model, the proposed Virtual Historic Dublin City will improve the current approaches for planning, conserving, presenting and interpreting cultural heritage buildings and their environments. The combination of digital recording, modelling and data management systems enable the interaction with complex, interlinked three-dimensional structures containing rich and diverse underlying data. End users can encompass architectural and engineering conservation, education and research, in addition to public engagement and cultural tourism. In this paper a digital design framework is presented, based on state of the art current approaches for recording, modelling and presenting Virtual Historic Dublin. The modelling sites and structures is based on remotely sensed survey data which is processed and modelled in Historic BIM or GIS allowing the addition of semantic attributes. Archiving and storage of both models and knowledge and information attributes requires open systems and server data base capable of handling vector and point cloud information in addition to other digital data. The dissemination and interaction with the models and attached knowledge attributions is based on combining game engine platforms, Historic BIM, Historic GIS and access to compatible storage and data base.
\end{abstract}

\section{INTRODUCTION}

Historic rural, urban or town centres however large or modest in scale include human memory within their buildings, environments and objects and are a current but fragmented focus for global digital recording, virtual modelling and archiving. As an endeavour to digitally assemble these connected tangible and intangible cultural assets the Virtual Historic Dublin is suggested as a dynamic digital repository and portal. This represents a move from purely object visualisation to intelligent digital representation which will allow multiple user scenarios ranging from conservation to education and knowledge extraction. The combination of digital recording, modelling and data management systems enable the interaction with complex, interlinked three-dimensional structures containing rich and diverse underlying data. End users can encompass architectural and engineering conservation, education and research, in addition to public engagement and cultural tourism. It is essential to incorporate within a design framework international principles concerning authenticity, integrity and philosophical approaches such as those promoted in ICOMOS Charters and UNESCO Recommendations.

The potential to generate intelligent virtual models of historic buildings and their environments has greatly improved and is mainly due to the advances in data capturing technologies like laser scanning and new methods for 3D modelling of historic buildings (BIM and GIS). A new application of these technologies is proposed for constructing Virtual Historic Dublin as an integral part of the smart city to integrate information from different scales, sources and disciplines in a single, coherent model. Figure 1 below, illustrates the main elements (a.) point cloud of historic centre of Dublin, (b) sample historic map and (c) 3D models of historic structures.

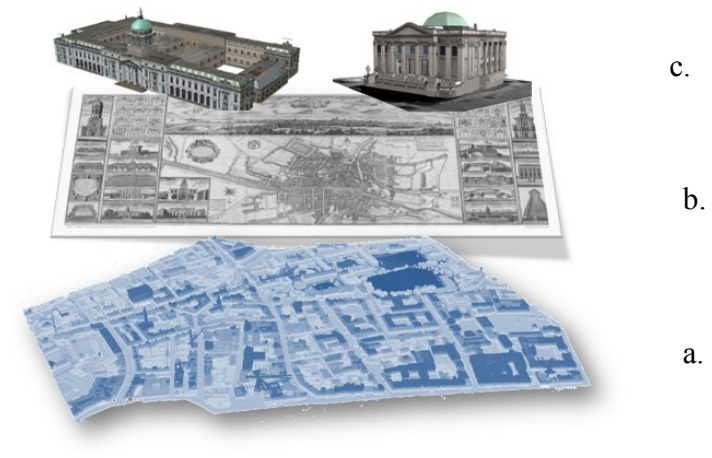

Figure 1: Dublin City Aerial Scan, Historic Maps and 3D Models

As a single coherent model, the proposed Virtual Historic Dublin City will improve the current approaches for planning, conserving, presenting and interpreting cultural heritage buildings and their environments.

In this paper a digital design framework is presented, based on (a) modelling sites and structures, (b) archiving and storage and (c) dissemination based on game engine platforms, HBIM and HGIS.

\section{3D MODELLING}

\subsection{Evolution}

3D modelling concepts have developed from early 2D and 3D CAD representations which were mainly composed of $2 \mathrm{D}$ or $3 \mathrm{D}$ lines. The first $3 \mathrm{D}$ wireframe models were an extension of $2 \mathrm{D}$ 
drafting where 3D lines were manually drawn to create a 3D wireframe model. Two important advancements in 3D CAD modelling introduced in the 1970s and 1980s were the concepts Constructive Solid Geometry (CSG) and Boundary Representation (BREP). CSG uses solid primitive shapes to represent objects. Solid objects can be used to calculate various physical properties such as volume, density, weight and mass. CSG also allows solid primitive shapes to be combined using Boolean operations such as union, subtract and intersect to create more complicated shapes. Operations and transformations in 3D modelling platforms include extrude, sweep and revolve which can be used to create 3D shapes from 2D outlines, this is illustrated in Figure 2

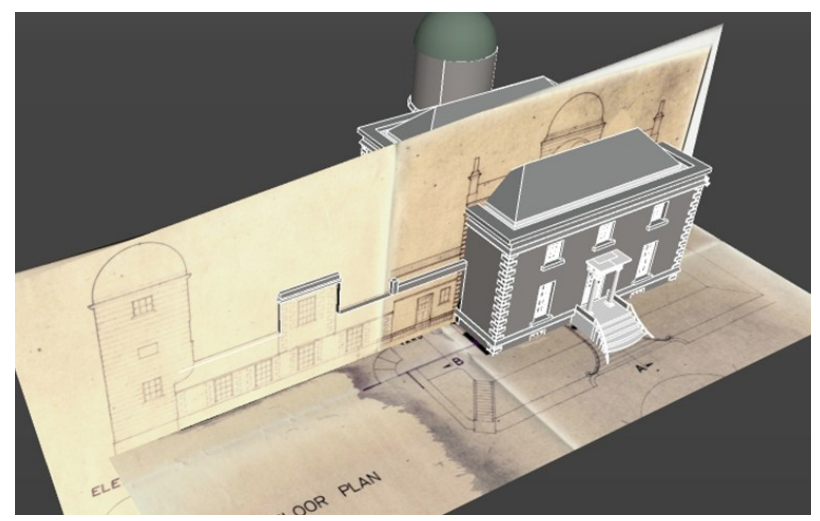

Figure 2: Building extruded from plans and elevations

Alternatively, Boundary Representation (BREP) represents objects by describing their faces, edges, vertices and topology.

It is evident that simple extrusions for historic building elements will not reflect the accuracy of deformations and nonuniform geometry of historic structures. There is therefore a need to categorise and connect the level of detail and the generative process to the accuracy and the authenticity required for the complete model (Banfi, 2017)

The next evolutionary stage in 3D modelling was the introduction of parametric and feature-based modelling which introduced a certain amount of intelligence into model elements. Feature-based modelling is an object orientated approach where in addition to geometry, objects contain information about the objects role (e.g. door, wall, window etc.) and how an object relates to other objects.

\subsection{Parametric Modelling and BIM}

Parametric modelling differs from standard 3D CAD modelling as objects such as primitive shapes are associated with parameters or variables that can instantly change the geometry or other properties of an object. The figure below illustrates the initial extruded rafter which is combined with other elements to build the hipped roof each element can be changed in shape position to create different iterations for many types of roofs.

Simple parameters of an object may include an object's length, width, height or radius. Other more complex parametric objects may have parameters that can change the entire structure or geometry of an object depending on different conditions. Parameters of an object can also control the location of an object within a larger model. Parametric library objects (such as doors or windows) allow objects to be reused multiple times in a model or in many different models with varying parameters. This approach is very efficient for modelling elements that are repeated but may contain geometric variation between different instances.

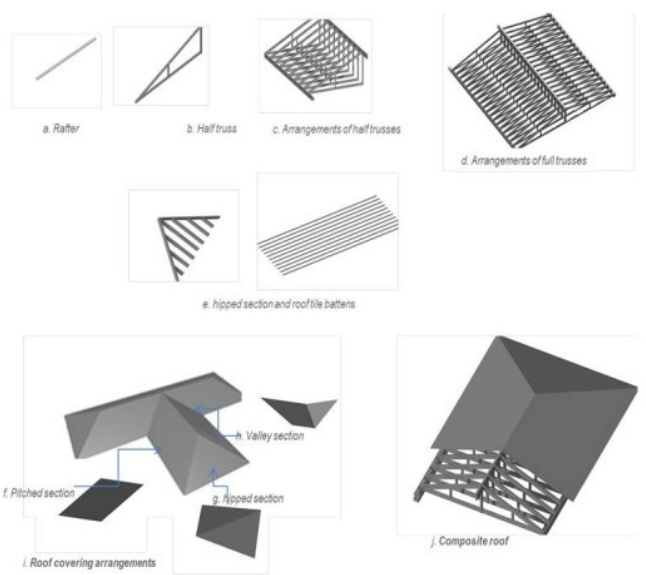

Figure 3: Parametric Roof Model

The more recent development of the concept Building Information Modelling (BIM) incorporates the main developments in 3D modelling including parametric and feature-based modelling combined with a dynamic 3D database for storing information relating to buildings. The addition of a dynamic relational database for building elements (similar to a Geographic Information System) enables many new applications for managing and analysing building elements. BIM enables building elements to be documented with smart parametric reusable objects that contain rich information about the objects use, semantics, topology, relationships with other objects and further information stored as attributes. BIM can be defined as the assembling of parametric objects which represent building components within a virtual environment and which are used to create or represent an entire building. Objects are described according to parameters some of which are userdefined and others, which relate to its position in a 3D environment relative to other shape objects. The visualisation of objects is achieved through viewing $2 \mathrm{D}$ and $3 \mathrm{D}$ features, plans, sections, elevations and 3D views. BIM can automatically create cut-sections, elevations, details and schedules in addition to orthographic projections and $3 \mathrm{D}$ models (wireframe or textured and animated. All of these views are linked to the 3D model and automatically update in real time so if a change is made in one view, all other views are also updated. This enables fast generation of detailed documentation required in the AEC/FM and heritage industries (Dore and Murphy, 2017).

\subsection{Historic Building Information Modelling (HBIM) and Heritage GIS}

By adding information and knowledge as semantic attributes to remotely sensed survey data, this then moves digital objects from static representations to dynamic, interactive and 'smart' models. Historic Building Information Modelling (HBIM) and Heritage GIS encompass the automated data capture of existing structures and sites followed by mapping of parametric and information rich objects onto a geometric framework developed from survey data. The resultant Historic BIM or HGIS can then be used for information and knowledge extraction in addition to visualisation. As a multi-disciplinary and evolving system, researchers from computing, architecture, archaeology, 
engineering and other heritage areas are improving the process for efficiency and for creating more open systems. Software efficiency is based around automatic object recognition in point clouds and semiautomatic procedural modelling approaches. The development of open systems emphases lower cost and open source platforms in contrast to existing expensive commercial platforms.
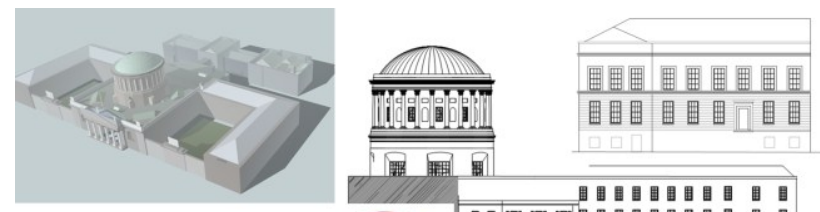
因田目目目田畐目目
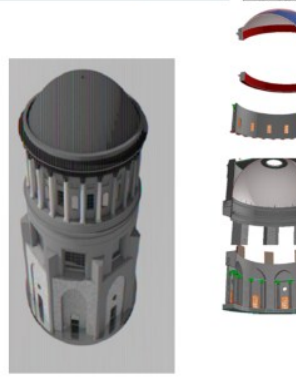

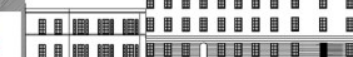
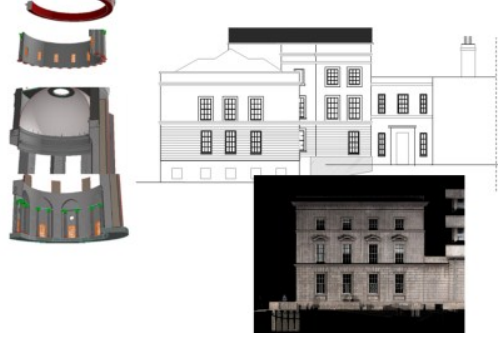

Figure 4: HBIM of Dublin Four Courts and sample conservation documentation automatically generated from the HBIM

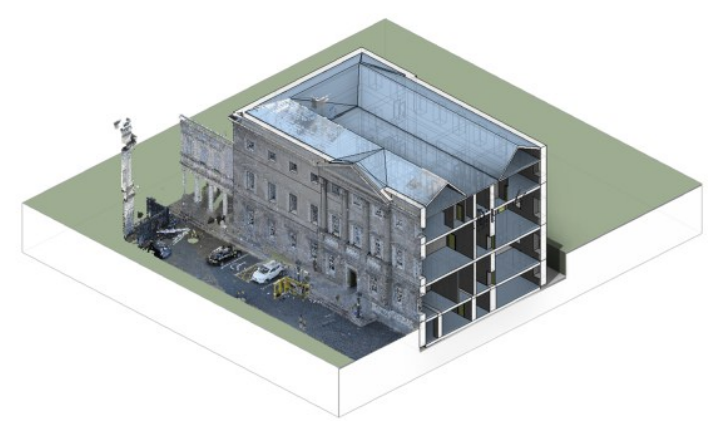

Figure 5: A combination of BIM and Scan for Leinster House Irish Parliament Building

\subsection{Parametric Libraries and Shape Grammars}

Architectural elements as 3D objects are mapped onto the point clouds surveys. The design for creating these objects as illustrated in the Figure below, is based on architectural rules informing shape grammars which are transformed from primitive to complex shapes based on operations such as add/subtract, rotate, deform, extrude etc. The shapes are then arranged to fit the form of historic structures or sites (Murphy et al 2013).

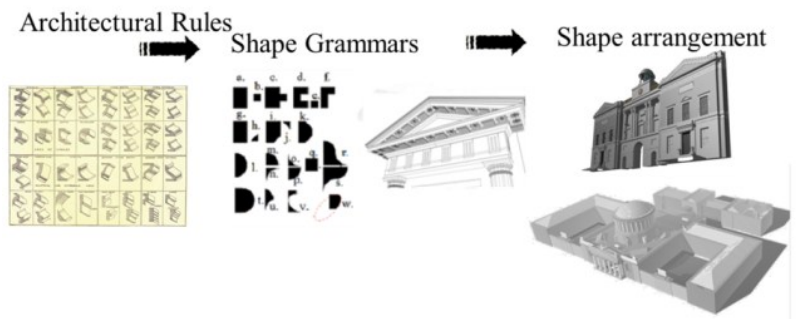

Figure 6: design for HBIM library of architectural elements

\subsubsection{Shape Rules}

For intelligent computer information modelling of architecture, the composition of a building can be describes as a formulated set of rules which govern the distribution and combination of building parts or elements (walls, floors, roofs, windows, ornament etc.) within a 3D space. In addition buildings are also based on different architectural styles which determine the rules for combing these elements. Described as a shape grammar the building can be divided up and represented by sets of basic shapes which are a limited arrangement of straight lines in three-dimensional Euclidian space. These shapes are governed by replacement rules whereby a shape can be changed or replaced by transformations and deformations and also (Stiny 1980).

\subsection{Procedural Modelling}

A major challenge in the generation of high quality $3 \mathrm{D}$ models of existing buildings is the high modelling/conversion effort and cost required for creating semantic building models from unstructured captured building data (point clouds). Other challenges include the difficulties in accurately representing the variety of complex and irregular objects occurring in existing buildings and the lack of standards for the representation of objects and information in existing buildings (Volk et al., 2014). The automatic generation of structured building information models from point clouds is a primary focus for a lot of research in this area. Jung et al. (2014), Xiong et al. (2013) and Zhang \& Zakhor (2014) have shown promising results for automatic object recognition and feature extraction from point cloud data. Although progress has been made in this area, results are currently limited to automatic extraction of basic elements such as planes and openings. Automatic extraction of complex architectural elements that occur in existing and historical buildings is still in its infancy. The results from existing automatic approaches include surface models, planes, 3D vectors or a subset of the original point cloud. All of these results still need to be converted into structured information enhanced and parametric building components which at present needs to be carried out manually (Volk et al., 2014). A procedural modelling approach uses a sequence of generation instructions, rules or algorithms that can be repeated with varying characteristics to automatically generate 3D geometries (Kelly \& Mccabe, 2006). Due to its efficiency and speed of modelling, procedural modelling approaches are now also being applied for modelling existing buildings (Muller et al., 2006, Hohmann et al., 2009 and Thaller et al., 2011, Dore and Murphy 2017). 


\section{VIRTUAL HISTORIC DUBLIN}

\subsection{Lidar Scan of Historic Centre of Dublin}

The 2015 Aerial Laser and Photogrammetry Survey of Dublin City Collection Record will be used to develop the Virtual Historic City of Dublin, developed from existing data sets of historic 2D mapping and in particular aerial and terrestrial laser and image based surveys. The Lidar and 3D mapping surveys were carried by the School of Engineering UCD in 2008 and 2015 funded by Science Foundation of Ireland and European Research Council. The survey data includes most of Dublin City's medieval and classical historic areas. The high density, aerial remote sensing data for a $2 \mathrm{~km}^{2}$ area of Dublin, Ireland obtained at an average flying altitude of $300 \mathrm{~m}$. The data include aerial laser scanning (ALS) from 41 flight paths in the form of a 3D point-cloud (LAZ) and 3D full waveform ALS (LAS and Pulsewave). Imagery data includes ortho-rectified 2D rasters (RGBi) and oblique images. The ALS data consist of over 1.4 billion points. The ALS and imagery data are structured both by flight paths and by $500 \times 500 \mathrm{~m}$ rectangular tiles (Laefer, D, F, et al 2018).

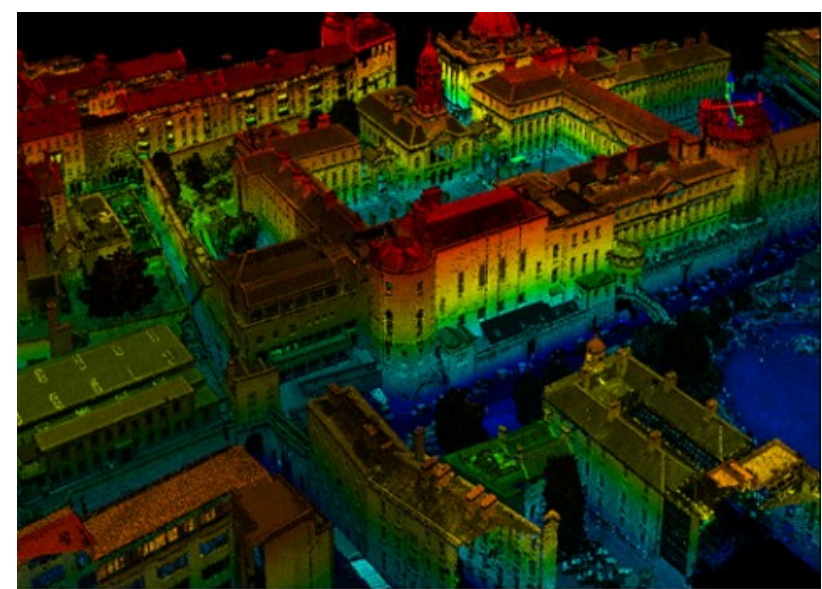

Figure 7: Sample of Aerial Laser and Photogrammetry Survey of Dublin Historic City

\subsubsection{Processing the Lidar Data Sets}

In addition to solid 3D CAD or BIM models meshing of point clouds can either create whole or part models or used to enhance the HBIM models and achieve real world geometry. Polygonal surface meshing creates a surface on a point cloud; the created surface is made up of triangles connecting the data points into a consistent polygonal model. A mesh is defined as a collection of triangular (or quadrilateral) contiguous, nonoverlapping faces joined together along their edges which contain vertices, edges and faces forming the representation of the face of an object. The point cloud, is a series of random points, which are then linked by triangular networks using algorithms such as Delaunay triangulation. The meshing algorithm simultaneously identifies on the point cloud, the vertex of each triangle that lies within the interior of any of the circum-circles of each triangle. The triangles can then be surfaced. The function of smoothing modifies the surface structure of the point cloud by optimising the point data, filling holes and correcting edges. This is followed by decimation which is a process to optimise the number of polygons and points in the mesh.
This point cloud data is at present segmented manually and converted to meshes for importation into HBIM, Game Engine and GIS platforms. Automatic segmentation and meshing will be considered as proposed by Ozdemir and Remondino (2018). In the following series of steps the point cloud after merging, cropping and cleaning was imported into Geomagic Wrap (Step 3 ), a sample rate of $100 \%$ of the point cloud ensures none of the scan is removed and also data is imported in the correct units. Max triangles are set so the mesh does not use up to much memory and be unusable. To clean the Mesh a combination of automatic and manual hole filling is carried out and using flat fill options. Mesh Doctor is used for final cleaning and smooth out triangles.

Figure 14 illustrates a series of steps which follow illustrate some of the workflows involved for processing Lidar Data Sets as part of Virtual Historic Dublin.

STEP 1 Point cloud merged, cropped, and re-coloured
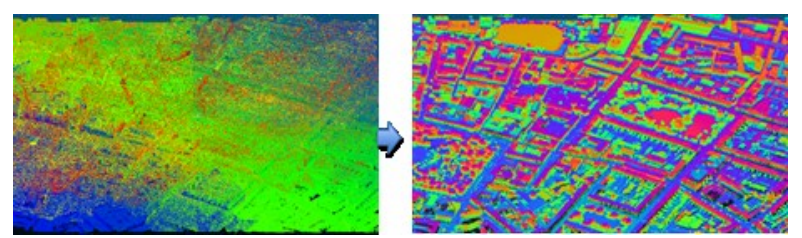

STEP 2 Convert to E57 format files for use in HBIM

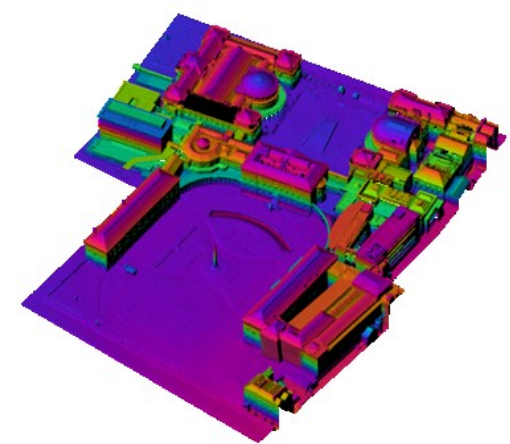

STEP 3 Geo-Magic Wrap and transformed into a mesh, cleaning to remove noise and fill holes in the data

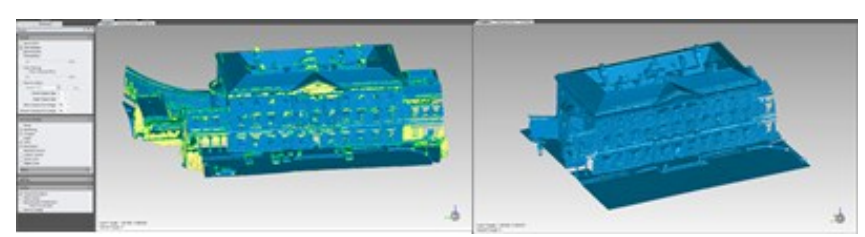

STEP 4 HBIM created from historic data, scan data and mesh 


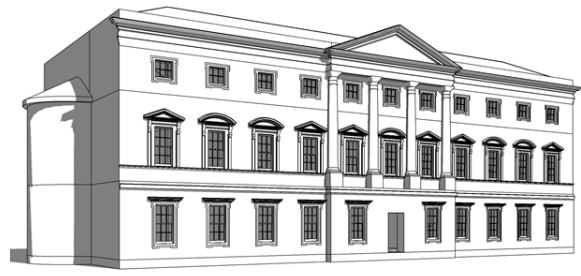

Figure 8: Initial Workflows Involved For Processing Lidar Data Sets to HBIM

\subsection{ARCHIVING AND STORAGE}

\subsection{Content -Knowledge and Information Attributes}

Virtual Historic Dublin has included in its design framework how archaeological and historic knowledge is produced and applied to enrich this project. This design framework focuses on digital data curation and presentation of archaeological and architectural heritage in Dublin's historic centres. This process involves maintaining, preserving, interpretation and adding value to information, knowledge concerning archaeological material and objects. The organised digital data is enriched with semantic attributes from varied sources and stored within a data base or repository linked to a 3D model of the Dublin's historic centre, allowing access for various end user scenarios.

By understanding how archaeologists, historians and other specialists create and use knowledge will determine how archaeological data and historic material collections can be added to Virtual Historic Dublin. The virtual replication of archaeological and architectural heritage structures is enhanced with the addition of knowledge and information content as semantic attributes to the model. By adding information and knowledge as semantic attributes to digital heritage models, this then moves digital objects from static representations to dynamic, interactive and 'smart' models. The resultant Smart Model can then be used for information and knowledge as a multi-disciplinary and evolving system.

The Definition of knowledge in the context of archaeological practices is accomplished through reviewing and researching sources related to surviving artefacts, structures, environments. An ethnographic understanding will be developed through rigorous exploration of these sources of data. Using these data sources as a foundation, a framework of analysis based on interviews and questionnaires will be developed with archaeologists and other experts in order to collect their understanding. The design of end user scenarios will be implemented for understanding and predicting how virtual models can be employed to disseminate attributed knowledge content in the context of Virtual Historic Dublin.

\subsection{Data Curation}

Digital data curation involves maintaining, preserving, interpretation and adding value to information, knowledge, material and objects. This includes activities related to organisation, the annotation of data and systems to ensure survival of data collected from various sources. Quality systems ensure that content is authentic, of undisputed origin and genuine. Figure 9, outlines the information flow for Virtual Historic Dublin.
A management system safeguards lasting value and lessens the risk of digital obsolescence. Open repositories ensure that curated data not only survives but is shared with wider communities for their use and enhancement avoiding duplication of effort. The initial workflow for this model starts with the capture of data followed by its classification and organisation. The organised data is then enriched with semantic attributes from other sources and stored within a data base or repository allowing access for various end user scenarios. The entire work flow is continuously updated and improved through a continuing conceptualisation, planning and evaluation process and managed to ensure quality and survival of data.

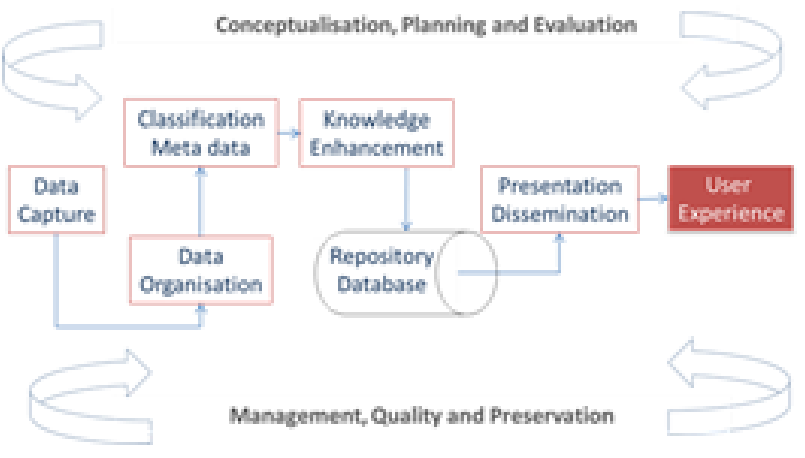

Figure 9: System Architecture for Archival and Storage Repository

\subsection{DISSEMINATION BASED ON GAME ENGINE PLATFORMS}

Game engine platforms allow a low-cost method of making intelligent models and linked data more easily accessible to users. It is the nature of interactive videogame applications to be intuitive to the user quickly upon assuming controls. A packaged 'game file' is designed to execute in a standalone fashion, requiring no additional proprietary software installed on the end-users computer system. Current mainstream industry packages include Unity3D and the Unreal Engine which allow for highly developed workflows and community support, but recent game engine software like Autodesk's Stingray package hold promise for greater interoperability with BIM.

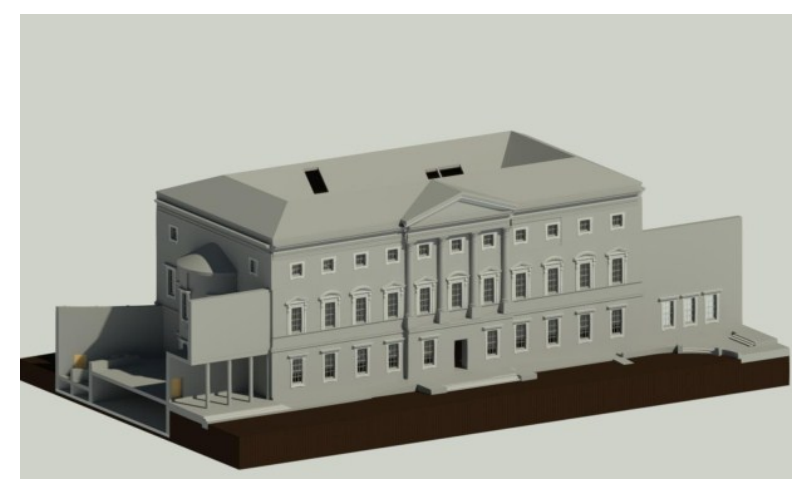

Figure 10: HBIM model imported into Unity Game Engine Platform 
With regards to educational applications, game engines can give public access to information that is usually restricted to specialists The final stage of the workflow involves linking the game engine based Historic BIM to a semantic web platform. This enables end-users to interact with the virtual building and to access the rich data related to the model without needing to install specialist BIM software. Shape, geometry and geolocation can be linked to enriched data in the model and held externally. User queries can be linked to the locations of elements in the building, to shapes (such as design features) and to the semantics of the information, and will be facilitated by data flow between the game engine, 3D HBIM components server and data stores. The delivery options for the Virtual Historic City range from WEB based and VR immersion to Augmented Reality.

\section{CONCLUSIONS}

\subsection{Systems Architecture}

The initial design framework for Virtual Historic Dublin is presented in Figure 10, below. Stage 1, illustrates the input data ranging from historic to laser scan and other survey data. This data is then processed and enriched with knowledge and information attributes (Stage 2) but also can be used in raw state. There are a series of data base servers the first is the historic components as 3D HBIM which maintains the libraries of intelligent objects which represent the elements of a building structure. BIM authoring platforms are mostly tailored for modern architecture and their libraries of parametric architectural elements/objects are limited to basic components. To overcome this problem a new design model is applied using architectural rules and shape grammars to code primitive and complex historic architectural objects (Hichri et al, 2014; Murphy et al, 2013; Santagati and Turco, M, 2017). The architectural objects are mapped onto a geometric framework made up of point cloud, image data and historic digital surveys (Stagel 3). A server is dedicated to game engine assets and the system can also be linked into external data bases.

The model at stage 3 hols AEC information and is then enhanced with geo-location, with the exception of the 3D HBIM components server (Detail 2) this can be considered a standard process pipeline. While BIM platforms have the potential to create a virtual and intelligent representation of a building, its full exploitation and use is restricted to narrow set of expert users with access to costly hardware, software and skills. In the final stage, the semantically enriched model is transferred into a WEB based game engine platform. This enables not only interaction with the virtual building but allows users to access and query related information rich data contained in the model and externally. The user access and queries are linked to the geo-location of elements of the building and to geometry shape and attributed semantics facilitated by data flow between the game engine asset server (Detail 4) and the 3D HBIM components server (Detail 2).

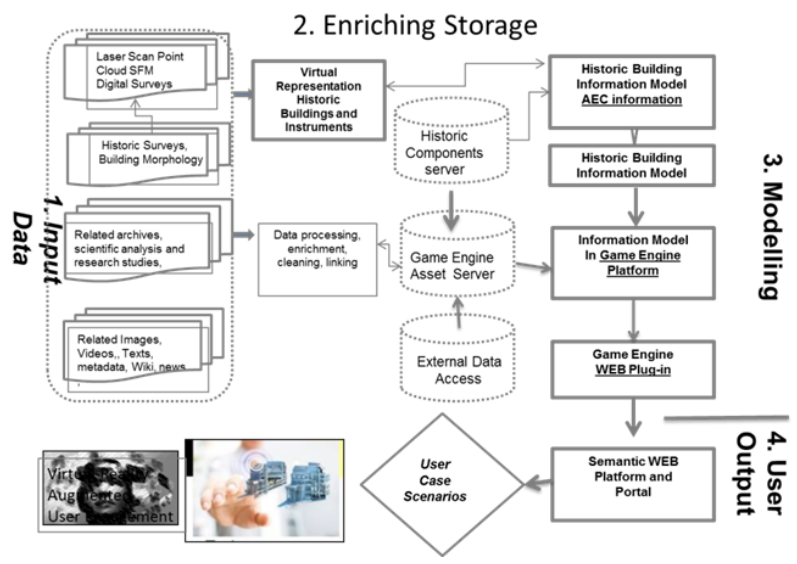

Figure 11: Systems Architecture for Dissemination

\subsection{Data Base - PostGreSQL/PostGIS}

Oracle and Postgre SQL spatial databases are proposed for managing large datasets. The storage of spatial data in addition to GIS Spatial data sets are primarily defined as those which are directly or indirectly referenced to a location on the surface of the earth and non-spatial data (text, image etc.). Spatial data bases now include vector data which include three geometric primitive types and geometries; 2-D Point and Point Cluster, 2D Line Strings, 2-D N-Point Polygons. PostgreSQL is an open source, object-relational database management system which can be extended to handle $2 \mathrm{D}$ and $3 \mathrm{D}$ spatial data using an extension called PostGIS (Obe and Hsu, 2011). However, in order to import SketchUp models into PostGIS, a CityGML file must be created from the SketchUp model and a software called 3DCityDB is required to transfer CityGML into PostgreSQL/PostGIS.

\section{REFERENCES}

Constantopoulos, P., Dallas, C., Androutsopoulos, I., Angelis, S., Deligiannakis, A.,Gavrilis, D., et al. (2009). DCC\&U: An extended digital curation lifecycle model. International Journal of Digital Curation, 4(1), 34-45.

Dore, C.; Murphy, M. Current State of the Art Historic Building Information Modelling. Int. Arch. Photogramm. Remote Sens. Spat. Inf. Sci. 2017, 42, 185-192.

Edwards, Gareth, Haijiang Li, and Bin Wang. "BIM based collaborative and interactive design process using computer game engine for general end-users." Visualization in Engineering 3, no. 1 (2015): 4.

Hichri N., Stefani C., Veron P., Hamon G., De Luca L. Review of the «as-built» BIM approaches. Journal of Applied Geomatics. Springer Berlin / Heidelberg, 2014

Hohmann, B, Krispel, U, Havemann, S \& Fellner, D 2009, 'CityFit - High-quality urban reconstructions by fitting shape grammars to images and derived textured point clouds', paper presented to 3DARCH 2009 - 3D Virtual Reconstruction and Visualization of Complex Architectures, Trento, Italy, 25-28 February 2009. 
Huvila, I., Huggett, J. (2018). Archaeological Practices, Knowledge Work and Digitalisation. Journal of Computer Applications in Archaeology, 1(1): 88-100

Jung, J, Hong, S, Jeong, S, Kim, S, Cho, H, Hong, S \& Heo, J 2014, 'Productive modeling for development of as-built BIM of existing indoor structures', Automation in Construction, vol. 42, no.0, pp. 68-77.

Lauzikas, R., Dallas, C., Thomas, S., Kelpšiene, I., Huvila, I. et al. (2018). Archaeological Knowledge Production and Global Communities: Boundaries and Structure of the Field. Open Archaeology, 4(1): 350-364

Kelly, G \& Mccabe, H 2006, 'A Survey of Procedural Techniques for City Generation', ITB Journal, no.14, pp. 87130.

Laefer, D. F., Abuwarda, S., Vu Vo, A., Truong-Hong, L. and Gharibi H., 2015. Aerial Laser and Photogrammetry Survey of Dublin City Collection RecordNew York University. Center for Urban Science and Progress, doi:10.17609/N8MQ0N, https://geo.nyu.edu/?f\%5Bdct_isPartOf_sm $\% 5 \mathrm{D} \% 5 \mathrm{~B} \% 5 \mathrm{D}=201$ 5+Dublin+LiDAR, (Last Accessed December 2018)

Muller, P, Wonka, P, Haegler, S, Ulmer, A \& Gool, LV 2006, 'Procedural Modeling of Buildings', ACM Transactions on Graphics, vol. 25, pp. 614-23.

Murphy, M, McGovern, E \& Pavia, S 2013, 'Historic Building Information Modelling - Adding intelligence to laser and image based surveys of European classical architecture', ISPRS Journal of Photogrammetry \& Remote Sensing, vol. 76, pp. 89102.

Oreni, D.; Brumana, R.; Georgopoulos, A.; Cuca, B. Hbim for Conservation and Management of Built Heritage: Towards a Library of Vaults and Wooden Bean Floors. ISPRS Ann. Photogramm. Remote Sens. Spat. Inf. Sci. 2013, II-5/W1, 215221.

Parish, YIH \& Müller, P 2001, 'Procedural Modeling of Cities', paper presented to 28th annual conference on Computer graphics and interactive techniques, New York, 2001.
Thaller, W, Krispel, U, Havemann, S, Redi, I, Redi, A \& Fellner, DW 2011, 'Developing Parametric Building Models The Gandis Use Case', paper presented to 4th International Workshop 3D-ARCH 2011, "3D Virtual Reconstruction and

Santagati, C, Turco, M, 2017, From structure from motion to historical building information modelling: populating a semantic-aware library of architectural elements, Journal of Electronic Imaging, Volume26, International Society for Optics and Photonics, pp 011007-011007.Visualisation of Complex Architectures", Trento, Italy, 2-4 March.

Stiny, G 1980. Introduction to shape and shape grammars. Environment and Planning B: Planning and Design 7 343-351

Volk, R, Stengel, J \& Schultmann, F 2014, 'Building Information Modeling (BIM) for existing buildings Literature review and future needs', Automation in Construction, vol. 38, no. 0, pp. 109-27.

Xiong, X, Adan, A, Akinci, B \& Huber, D 2013, 'Automatic creation of semantically rich $3 \mathrm{D}$ buildingmodels from laser scanner data', Automation in Construction, vol. 31, no. 0, pp. 325-37.

Yan, Wei, and Geqing Liu. "BIMGame: Integrating building information modeling and games to enhance sustainable design and education." In Predicting the Future [25th eCAADe Conference Proceedings] Frankfurt am Main (Germany), pp. 211-218. 2007.

Zhang, R \& Zakhor, A 2014, 'Automatic identification of window regions on indoor point clouds usingLiDAR and cameras', paper presented to Applications of Computer Vision (WACV), 2014 IEEE Winter Conference on, 24-26 March 2014.

\section{ACKNOWLEDGEMENTS}

Acknowledgements of support for the project from OPW Ireland and COST ARKWORK 